\title{
Measures on Projections and Physical States
}

\author{
Erik Christensen \\ Matematisk Institut, Københavns Universitet, DK-2100 København, Danmark
}

\begin{abstract}
It is shown that a finitely additive measure on the projections of a von Neumann algebra without $I_{2}$ and $I I_{1}$ summands is the restriction of a state. A definition of a physical state is proposed, and it is shown that such a physical state on a simple $C^{*}$-algebra with unit is a state.
\end{abstract}

\section{Introduction}

In the classical mathematical model for quantum mechanics one has the notions of "yes-no experiment" and "state". The state of the system is a certain preparation of it, and one associates to each experiment a value in each state, namely the probability for yes in this state, and this meanvalue is then called the value of the state on the proposition. As a more detailed description of the set of propositions including their lattice properties, one uses operator algebras, and one supposes that the propositions correspond to projections in a noncommutative self-adjoint algebra of operators on a Hilbert-space $[5,6]$. The value of the state on the union of two compatible disjoint propositions is the sum of the values on each, and since disjoint compatible propositions correspond to pairwise orthogonal projections, we find that the state is transformed into a function on the set of projections in the algebra, which is additive on any finite set of pairwise orthogonal projections. Such a function is from now on called a measure on projections.

In [6] Mackey asked whether a countably additive measure is the restriction of a positive linear functional on the algebra - just as in ordinary integration theory and shortly after, Gleason [3] settled the question in the affirmative for the algebra of all bounded operators on a Hilbert space of dimension at least three. Various people have worked on the problem and we want to mention the works by Aarnes [1] and Gunson [4], upon which this paper is built. Gunson proves that countably additive measures on hyperfinite $I I_{1}$ factors are restrictions of states, by using showing that such measures have certain continuity properties, and then Gleason's result on the dense algebra consisting of a union of an increasing sequence of finite dimensional subfactors. The fundamental lemma Gunson uses is stated in Lemma 2.3 and it is also crucial here. Aarnes does not study the measure 
problem directly, but the similar problem in the $C^{*}$-model for quantummechanics. In this model one assumes the physical state to be a function on the algebra which is a positive linear functional when restricted to self-adjoint abelian subalgebras. From Aarnes' work we use especially his investigations on pure physical states and their relations to the primitive ideal spectrum of the algebra.

In Sect. 2 of the paper we introduce some notation and we discuss Gunson's Lemma.

In Sect. 3 we show that physical states on certain fields of algebras of compact operators on locally compact spaces are always linear. In particular this covers the finitely additive measure question for type $I$ von Neumann algebras without central $I_{2}$ summands. This result, in turn, is then used in Sect. 4 to prove the similar result for properly infinite von Neumann algebras. At the end in Sect. 5 we propose a new definition for physical states and we dare to try to justify it by nonmathematical arguments. After the definition we show that such a physical state on a simple $C^{*}$-algebra with unit is linear.

\section{Notation and Gunson's Lemma}

As basic references to operator algebras we use the books by Dixmier [2] and Pedersen [8]. We will let $H, K$ denote Hilbert spaces, small Greek letters denote vectors in such spaces, $\mathscr{A}, \mathscr{B}$ are used for $C^{*}$-algebras, $M$ and $\hat{M}$ are names for von Neumann algebras and small Roman letters correspond to operators. We use $B(H)$ as a symbol for all bounded operators on $H$ and $C(H)$ for all compact operators.

2.1. Definitions. A measure on a $C^{*}$-algebra is a positive function on the set of projections which is additive on orthogonal projections. A measure $\mu$ is said to be a probability measure if $\mu(I)=1$. A quasi-state on a $C^{*}$-algebra $\mathscr{A}$ is a function $\varrho: \mathscr{A} \rightarrow \mathbb{C}$ such that

i) $\varrho$ is a positive functional on each abelian $C^{*}$-subalgebra.

ii) $\varrho(a+i b)=\varrho(a)+i \varrho(b) ; a=a^{*}, b=b^{*}$.

iii) $\sup \left\{\varrho(a) \mid a \in \mathscr{A}_{1}^{+}\right\}=1$.

By [1, Proposition 1, p. 607] there is a one-to-one correspondence between quasi-states and probability measures on von Neumann-algebras. The correspondence is given by

$$
\hat{\mu}(a)=\int_{\sigma(a)} \lambda d \mu\left(e_{\lambda}\right) ; \quad \mu=\hat{\mu} \mid\{\text { projections }\},
$$

where $e_{\lambda}$ is the spectral resolution of a self-adjoint element $a$.

In order to state Gunson's Lemma we need the following definition.

2.2. Definition. Two projections $e$ and $f$ in a $C^{*}$-algebra $\mathscr{A}$ are said to be isoclinic, if there is an angle $\alpha \in\left[0, \frac{\pi}{2}\left[\right.\right.$ such that $e f e=\cos ^{2}(\alpha) e$ and $f e f=\cos ^{2}(\alpha) f$.

It is fairly easy to see that $e$ and $f$ are isoclinic if and only if they generate a copy of $M_{2}$ (the $2 \times 2$ matrices over $\mathbb{C}$ ). With this interpretation of isoclinic projections, we can get an idea of the role played by the following lemma, because it makes it possible for two rather general projections $f$ and $g$ with $\|f-g\|<1$ to 
find a third projection $h$ such that $f$ and $h$ are contained in a copy of $M_{3}, h$, and $g$ are contained in another copy of $M_{3}$, and $h$ is close to both $e$ and $f$, if they are close to each other. Knowing Gleason's result for $M_{3}$, one then gets the norm continuity of any probability measure on projections in $\mathscr{A}$.

2.3. Lemma. Let $f$ and $g$ be two projections in a $C^{*}$-algebra $\mathscr{A}$, such that $\|f-g\|<1$, and suppose there exists a partial isometry $u$ with $u^{*} u=f$ and $f u=g u=0$, then there exists a projection $h$ which is isoclinic with angle $\alpha=\frac{1}{2} \sin ^{-1}\left(\left\|(f(f-g) f)^{1 / 2}\right\|\right)$ to both $f$ and $g$.

Proof [4, Lemma 2.6, p. 299]. It suffices to see that the numbers $\alpha_{1}, \alpha_{2}$ defined by $\alpha_{1}=\alpha_{2}=\alpha$ satisfy

$$
\alpha_{1}+\alpha_{2}<\frac{\pi}{2}, \quad\left|\alpha_{1}-\alpha_{2}\right| f \leqq \sin ^{-1}\left((f(f-g) f)^{1 / 2}\right) \leqq\left(\alpha_{1}+\alpha_{2}\right) f
$$

but $\left\|\sin ^{-1}\left((f(f-g) f)^{1 / 2}\right)\right\|=\sin ^{-1}\left(\left\|(f(f-g) f)^{1 / 2}\right\|\right)$ since $\sin ^{-1}$ is increasing on $[0,1]$.

Gunson's proof then takes over with only a few modifications; it is for instance necessary to let $\cos \theta$ denote $(f g f)^{1 / 2}$ instead of $(I-f)+(f g f)^{1 / 2}$. Another point which needs a word is the relation (2.14) in Gunson's proof. At first hand it is not clear that the operator $B$ defined by $(2.14)$ belongs to the $C^{*}$-algebra generated by $f g f$, but $f-(f g f)^{1 / 2}=(f-f g f)\left(f+(f g f)^{1 / 2}\right)^{-1}$, and we find that the operator $B$ of $(2.14)$ is given by $(f-f g f)^{1 / 2}\left(f+(f g f)^{1 / 2}\right)^{-1} \cot \alpha$, which is easily seen to be a continuous function of $f g f$.

It is indicated above how Lemma 2.2 can be used to prove norm continuity, and this is also the way Gunson proves norm continuity, but he makes explicit use of the fact that he is dealing with a continuous von Neumann algebra, so we have to take a slightly different path.

2.4. Proposition. Let $f$ and $g$ be projections with $\|f-g\|<1$ in a $C^{*}$-algebra $\mathscr{A}, u$ and $v$ partial isometries in $\mathscr{A}$, such that $u^{*} u=f=v^{*} v, f u=g u=f v=g v=u^{*} v=0$, then for any probability measure $\mu$ on $\mathscr{A}$

$$
|\mu(f)-\mu(g)| \leqq 2\|f-g\|
$$

Proof. Construct via $f, g$, and $u$ a projection $h$ which is isoclinic to both $f$ and $g$ with angle $\alpha=\frac{1}{2} \sin ^{-1}\left(\|f(f-g) f\|^{1 / 2}\right)$. Then $f, g$, and $h$ are all orthogonal to $v v^{*}$, and we find that the two sets of partial isometries

$$
\left\{f,(\cos \alpha)^{-1} h f, v\right\} \text { and }\left\{g,(\cos \alpha)^{-1} h g, v g(g f g)^{-1 / 2}\right\}
$$

both generate type $I_{3}$ factors. Now $\hat{\mu}$ is linear on both these algebras and we get

$$
|\mu(f)-\mu(g)| \leqq|\hat{\mu}(f-h)|+|\hat{\mu}(h-g)| \leqq\|f-h\|+\|h-g\| .
$$

Clearly the norm of $(f-h)$ equals the norm or the $2 \times 2$ matrix

$$
\left(\begin{array}{cc}
1-\cos ^{2} \alpha & -\cos \alpha \sin \alpha \\
-\cos \alpha \sin \alpha & -\sin ^{2} \alpha
\end{array}\right)=\sin \alpha\left(\begin{array}{cc}
\sin \alpha & -\cos \alpha \\
-\cos \alpha & -\sin \alpha
\end{array}\right) \text {. }
$$


The matrix to the right is unitary, so

$$
\begin{aligned}
\|f-h\| & =\sin \alpha=\sin \left(\frac{1}{2} \sin ^{-1}\left(\|f(f-g) f\|^{1 / 2}\right)\right. \\
& \leqq\|f(f-g) f\|^{1 / 2} \\
& =\|(1-g) f\| \\
& \leqq\|f-g\|,
\end{aligned}
$$

and we get

$$
|\mu(f)-\mu(g)| \leqq 2\|f-g\|
$$

\section{Continuous Fields of Elementary Algebras}

By an elementary algebra we mean a $C^{*}$-algebra which is isomorphic to the compact operators on some Hilbert space. Continuous fields of elementary $C^{*}$-algebras are defined and studied in [3, Chap. 10]. Among other things it is proved that $C^{*}$-algebras with continuous trace in most cases are locally trivial continuous fields of elementary $C^{*}$-algebras on their spectrum.

3.1. Theorem. Let $T$ be a locally compact Hausdorff space, $A=((\mathscr{A}(t)), \Theta)$ a locally trivial continuous field of elementary $C^{*}$-algebras on $T$, and $\mathscr{A}=\Theta$ the $C^{*}$-algebra defined by $A$. If no $\mathscr{A}(t)$ is isomorphic to $M_{2}$, then all quasi-states on $\mathscr{A}$ are linear.

Proof. By [1, Proposition 2, p. 605] we may restrict our attention to pure quasistates. Let $\varrho$ be a pure quasi-state; from [1, Theorem 4, p. 612] we know that $\varrho$ is supported on a single point in $\hat{\mathscr{A}}$, the spectrum of $\mathscr{A}$, if this space is a Hausdorff space, but we know from [2, Corollaire 10.4.4] that $\hat{\mathscr{A}}$ is homeomorphic to $T$, so we find that $\varrho$ is concentrated in a single point $t_{0}$ of $T$. In order to prove that $\varrho$ is linear it is by [1, Corollary 2, p. 622] sufficient to prove that $\varrho$ is continuous.

We claimed that $\varrho$ is concentrated in $t_{0}$, but that is only true in the sense that we know $\varrho(x)=\varrho(y)$ if $x$ and $y$ agree in a neighbourhood of $t_{0}[1$, Lemma 2, p. 620], and what we actually are going to do is to show, that we may suppose, that we are dealing with functions which are locally constant around $t_{0}$, and we therefore can factor $\varrho$ through $t_{0}$. We have assumed local triviality so we will restrict our attention to the $C^{*}$-algebra $\mathscr{C}=C(U, C(H))$ where $U$ is a compact neighbourhood of $t_{0}$ and $C(H)$ is isomorphic to $\mathscr{A} / t_{0}$. Let $c$ be a positive operator in $\mathscr{C}$, then $c\left(t_{0}\right)$ is a compact operator and the spectrum of $c\left(t_{0}\right)$ consists of a decreasing sequence $\lambda_{1} \geqq \lambda_{2} \geqq$ such that $\inf \lambda_{n}=0$, if the sequence is infinite. Now $c$ is a continuous field, so in a sufficiently small neighbourhood of $t_{0}$ the operators $c(t)$ will have spectra which are close to the spectrum of $c\left(t_{0}\right)$. If $d$ is a self-adjoint close to $c$, then $d(t)$ is close to $c\left(t_{0}\right)$ in a certain neighbourhood and we do also find that the spectrum of $d(t)$ here is very similar to the spectrum of $c\left(t_{0}\right)$ in the meaning that the Hausdorff distances between the spectra is small. This shows that if our degree of accuracy is chosen in a suitable way, then to a given $\varepsilon>0$ we can find a continuous function $f$ with support in $\left[\varepsilon / 4, \infty\right.$ [, and a neighbourhood $V$ of $t_{0}$ such that for any $t$ in $V$

$$
\sigma(f(c)(t))=\sigma(f(d)(t))=\{0\} \cup\left\{\lambda_{i} \in \sigma\left(c\left(t_{0}\right)\right) \mid \lambda_{i}>\varepsilon / 4\right\} .
$$


Let $\lambda_{1}, \ldots, \lambda_{n}$ be the eigenvalues for $c\left(t_{0}\right)$ for which $\lambda_{i}>\varepsilon / 4$, and let $p_{j}, q_{j} \in C(V, C(H))$ be the spectral projections in this algebra corresponding to $\lambda_{j}$ for the operators $f(c) \mid V$ and $f(d) \mid V$. Clearly $p_{j}$ and $q_{j}$ can be supposed to be arbitrarily close to each other if one just first makes $V$ sufficiently small and secondly chooses $d$ close enough to $c$. We will therefore assume that

$$
\left\|p_{j}-q_{j}\right\| \leqq \varepsilon^{2}\left(4\left(1+\sum_{j=1}^{n} \lambda_{j}\right) \text { dimension } p_{j}\left(t_{0}\right)\right)^{-2} .
$$

It is fairly easy to see that we may assume that $\|c-f(c)\| \leqq \varepsilon / 4$ and $\|d-f(d)\| \leqq \varepsilon / 4$, so we obtain

$$
\begin{aligned}
|\varrho(c)-\varrho(d)| & \varrho(c-f(c))|+| \varrho(d-f(d))|+| \varrho(f(c))-\varrho(f(d)) \mid \\
& \leqq \varepsilon / 2+\left|\sum_{j=1}^{n} \lambda_{j}\left(\varrho\left(p_{j}\right)-\varrho\left(q_{j}\right)\right)\right| \\
& \leqq \varepsilon / 2+\sum_{j=1}^{n} \lambda_{j} \varrho\left(p_{j}\right)-\varrho\left(q_{j}\right) \mid .
\end{aligned}
$$

If the fibres are infinite dimensional, the conditions of Proposition 2.4 are fulfilled immediately. If the fibres are finite dimensional and not of dimension one, it may happen that the dimension of $p_{j}(t)$ is bigger than a third of the dimension of $H$. If this is the case we split $p_{j}$ into a sum of minimal projections $\left(r_{k}\right) k=1, \ldots \operatorname{dim} p_{j}\left(t_{0}\right)$ $\left(r_{k}\right.$ has support on all of $V$ ), and we find that $q_{j}$ can be split into a similar sum $\left(s_{k}\right)$ such that $\left\|r_{k}-s_{k}\right\| \leqq 2\left\|p_{j}-q_{j}\right\|^{1 / 2}$. We can now use Proposition 2.4 on $r_{k}$ and $s_{k}$ and we see from (1) and (2) that $\varrho$ is continuous, and the theorem follows.

\section{Von Neumann Algebras}

4.1. Theorem. Let $\mu$ be a probability measure on a von Neumann algebra $M$ without central summands of type $I_{2}$ or $I I_{1}$, then $\mu$ is the restriction of a state.

Proof. Section 3 covers the finite type $I$ part of $M$, so we may assume that $M$ is properly infinite.

The ingredient which is most important in this proof is, on the other hand, the result for finite type $I$ algebras. Suppose namely that $p$ and $q$ are two projections and suppose moreover that $(p \cup q)^{\prime} \cap M$ contains a copy, say $F$, of $M_{3}$ containing $I$, then $(p \cup q \cup F)^{\prime \prime}$ is a finite type $I$ von Neumann algebra with central summands of degree bigger than 3 , and the previous section shows that $\hat{\mu}$ is linear on $(p \cup q)^{\prime \prime}$.

Here we made assumptions on the size of the relative commutant, and we will in the rest of the proof fulfill this condition simply by looking at $\mu$ 's restriction to a subalgebra $\hat{M}$ of $M$, whose relative commutant is a factor of type $I_{3}$. When we have proven the linearity on $\hat{M}$, we claim that the reader can easily see that $\hat{\mu}$ will be linear on $M$ too. We will first show the existence of an increasing sequence $\left(p_{n}\right)$ of projections in $\hat{M}$ such that $p_{n} \sim\left(I-p_{n}\right) \sim I$ and $\mu\left(p_{n}\right) \geqq 1-2^{-n}$. We do this by induction, so assume that $p_{1}, \ldots, p_{k-1}$ are found, then $I-p_{k-1}$ can be split into a 
sum $f+g$ with $f \sim g \sim I$. Let $p_{k}$ be $p_{k-1}$ plus the one of the projections $f$ and $g$ on which $\mu$ takes the biggest value, then clearly $I \sim p_{k} \sim I-p_{k}$, and

$$
\mu\left(p_{k}\right) \geqq \mu\left(p_{k-1}\right)+\frac{1}{2}\left(1-\mu\left(p_{k-1}\right)\right)=\frac{1}{2}\left(1+\mu\left(p_{k-1}\right)\right) \geqq 1-2^{-k} .
$$

We will now show that $\hat{\mu}$ is linear on all the algebras of the form $p_{n} \hat{M} p_{n}$, by building any two positive small operators in such an algebra into two projections in $\hat{M}$. Let us fix $n$ and let $a$ and $b$ be two positive operators in $p_{n} \hat{M} p_{n}$ such that $0 \leqq a \leqq \frac{1}{2} p_{n}$ and $0 \leqq b \leqq \frac{1}{2} p_{n}$. In order to show that $\hat{\mu}(a+b)=\hat{\mu}(a)+\hat{\mu}(b)$, we construct two orthogonal projections $q$ and $r$ such that $p_{n} q p_{n}=a, p_{n} r p_{n}=b$, and $\hat{\mu}\left(p_{n} q p_{n}\right) \stackrel{\mathcal{E}}{\sim} \mu(q), \hat{\mu}\left(p_{n} r p_{n}\right) \stackrel{\varepsilon}{\sim} \mu(r)$. Let $\varepsilon>0$ be given and choose $k>n$ such that $\mu\left(p_{k}\right)>1$ $-\left(\frac{1}{6} \varepsilon\right)^{-2}$, moreover choose partial isometries $u, v$, and $w$ with common support $p_{n}$ and pairwise orthogonal ranges contained in $I-p_{k}$. Then the operators

$$
x=a^{1 / 2}+u a^{1 / 2}+v\left(p_{n}-2 a\right)^{1 / 2} \text { and } y=b^{1 / 2}-u b^{1 / 2}+w\left(p_{n}-2 b\right)^{1 / 2}
$$

are partial isometries with orthogonal ranges $q$ and $r$, satisfying the relations described above. For any of the projections $q, r$, and $q+r$ we have linearity on the algebra generated by that projection and $p_{n}$. Furthermore the range of $\left(I-p_{n}\right) q$ is contained in $\left(I-p_{k}\right)$, so by the Cauchy-Schwarz inequality we get for $q$

$$
|\mu(q)-\hat{\mu}(a)|=\left|\hat{\mu}\left(\left(I-p_{n}\right) q p_{n}\right)+\hat{\mu}\left(q\left(I-p_{n}\right)\right)\right| \leqq \frac{1}{3} \varepsilon,
$$

and also similar results for $r$ and $q+r$, so

$$
|\hat{\mu}(a+b)-\hat{\mu}(a)-\hat{\mu}(b)| \leqq \varepsilon .
$$

Define $f_{n}$ to be the linear functional on $\hat{M}$ given by

$$
f_{n}(x)=\hat{\mu}\left(p_{n} x p_{n}\right)
$$

then, since $\mu\left(I-p_{n}\right) \downarrow 0$, the sequence $\left(f_{n}\right)$ becomes a Cauchy sequence in the dual of $\hat{M}$ and hence converges to a state $f$ on $\hat{M}$. In order to complete the proof we show that the restriction of $f$ to the projections in $\hat{M}$ is $\mu$, so we look at an arbitrary $q$ and a $p_{n}$, and we find since both $\hat{\mu}$ and $f$ are linear on $\left(p_{n} \cup q\right)^{\prime \prime}$ and agree on $p_{n} \hat{M} p_{n}$, that

$$
|f(q)-\mu(q)| \leqq\left|f\left(p_{n} q p_{n}\right)-\hat{\mu}\left(p_{n} q p_{n}\right)\right|+\left|f(q)-f\left(p_{n} q p_{n}\right)\right|+\left|\mu(q)-\hat{\mu}\left(p_{n} q p_{n}\right)\right| .
$$

Since $f\left(p_{n}\right) \uparrow 1$ and $\mu\left(p_{n}\right) \uparrow 1$, we find $f(q)=\mu(q)$. In the beginning we claimed that it would not be a serious matter only to prove the theorem for $\hat{M}$ - a relative commutant of a type $I_{3}$ subfactor of $M$ - and we hope the reader is convinced when we mention that we can find a sequence of $I_{3}$ subfactors $F_{n}$ in such a way that there are sequences of projections $e_{n}$ and partial isometries $v_{n}, u_{n}$ such that $\left\{e_{n}, v_{n}, u_{n}\right\}$ belongs to $F_{n}, e_{n}$ is minimal in $F_{n}, v_{n}^{*} v_{n}=u_{n}^{*} u_{n}=e_{n}, e_{n}+v_{n} v_{n}^{*}+u_{n} u_{n}^{*}=I$, and $\mu\left(e_{n}\right) \uparrow 1$. So far we have proved that the functionals $g_{n}(x)=\hat{\mu}\left(e_{n} x e_{n}+v_{n} x v_{n}^{*}\right.$ $\left.+u_{n} x u_{n}^{*}\right)$ are all linear and we get that $g_{n}(p)$ converges to $\hat{\mu}(p)$ for each projection $p$, so $\hat{\mu}$ is linear.

\section{Physical States}

We have not been able to prove that all measures on projections in a von Neumann algebra of type $I I_{1}$ are restrictions of states, but we have, on the other hand, when trying to solve this problem, found some extra conditions which are 
sufficient. These conditions look reasonable from a physicist's point of view - we think - and they do give a result which carries over to quasi-states on simple $C^{*}$-algebras with unit.

We will first state our proposal for the definition of physical states and secondly try to convince the reader that this is reasonable for "physical" reasons.

5.1. Definition. A quasi-state on a $C^{*}$-algebra $\mathscr{A}$ is said to be a physical state if it belongs to the $w^{*}$-closed convex hull of states $\varphi$ which satisfy

1) $\operatorname{ker}(\varphi) \cap \mathscr{A}^{+} \neq\{0\}$ and $0 \leqq h \leqq k \in \operatorname{ker}(\varphi) \Rightarrow h \in \operatorname{ker} \varphi$.

2) For each $n$ in $\mathbb{N}$, there exists a quasi-state $\varphi_{n}$ on $\mathscr{A} \otimes M_{n}$, satisfying 1) and $\forall a \in \mathscr{A}: \varphi_{n}(a \otimes I)=\varphi(a)$.

The condition 1) is justified by the notion of state used by quantum physicists, e.g. Jauch. In [5, p. 92] Jauch describes a state as the result of a series of physical manipulations on the system which constitute the preparation of the state. We understand this in such a way that the manipulations are made, such that a number of physical data are fixed or known to be in certain regions. This means in the classical quantum mechanical model that certain questions have definite answers in this state and therefore that there exist non-trivial projections $p$ such that $\varphi(p)=0$. In the $C^{*}$-model for quantum mechanics we interpret this as the first half of condition 1). The second half may be more troublesome in general, but if we assume that operators have range projections inside the algebra, and that the physical state $\varphi$ is normal on abelian subalgebras, then for any pair $h, k$ with $0 \leqq h$ $\leqq k \in \operatorname{ker}(\varphi)$, the range projection $[k]$ belongs to $\operatorname{ker}(\varphi),[k]$ commutes with $h$ and dominates a positive multiple of $h$, so $\varphi(h)=0$, and we find that the second half is automatically fulfilled.

Let us now look at condition 2. For two compatible physical systems the algebra of observables for the combined system is the tensorproduct of the two algebras corresponding to each system, or perhaps more correctly; one assumes that the two algebras are commuting subalgebras of a third. Many of the algebras corresponding to physical systems contain copies of $M_{n}$, as an example one may look at an elementary particle in one dimension [5, Chap. 12]. The algebra corresponding to this system is normally considered to be all of $B(H)$, and therefore contains a copy of $M_{n}$, but if one does not want to include all these operators, one could instead look at the $C^{*}$-algebra generated by the spectral projections for the position-operator and the unitaries corresponding to space translations. At least for a mathematician who is out on thin ice, it looks reasonable that operators corresponding to such concrete things as place and translation should be in the algebra. Assuming this we find very easily a copy of $M_{n}$ in this algebra. Define namely $u$ to be the unitary which translates one unit length and define $e$ to be the spectral projection corresponding to the set

$$
\bigcup_{k \in \mathbb{Z}}\{[n k, n k+1]\}
$$

then one finds that $\left\{u^{i} e u^{* j} \mid 1 \leqq i, j \leqq n\right\}$, is a set of matrix units for a copy of $M_{n}$, containing $I$, inside this algebra of observables.

After this attempt to convince the reader that "real physical states" are physical according to this definition, we want to show that such states - at least in some cases - are linear. 
5.2. Theorem. Any physical state on a simple $C^{*}$-algebra with unit is linear.

Proof. It is enough to prove the statement for a quasistate, which satisfies 1) and 2) of definition 5.1 .

Let $\varphi$ be a physical state on the unital, simple $C^{*}$-algebra $\mathscr{A}$ and let $c$ and $d$ be elements in $\mathscr{A}$ such that $3 / 4 I \leqq c \leqq I$ and $3 / 4 I \leqq d \leqq I$. We will show linearity on $c+d$ by a method similar to the one used for properly infinite von Neumann algebras. To this end we first remark that for any $b$ in $\operatorname{ker}(\varphi) \cap \mathscr{A}^{+} \backslash\{0\}$ and any $a$ in $\mathscr{A}^{+}$, there exist elements $z_{1}, \ldots, z_{n}$ in $\mathscr{A}$, such that $a=\sum z_{i} z_{i}^{*}$ and $\sum z_{i}^{*} z_{i} \leqq N b$ for a suitable $N$ in $\mathbb{N}$. This follows from the Riesz-decomposition [7, p. 14], in the following way. Since $\mathscr{A}$ is simple with unit; $\mathscr{A}$ is also algebraically simple, and we find that $a=\sum^{n} s_{i}^{*} b t_{i}$, on the other hand $0 \leqq\left(s_{i}^{*}-t_{i}^{*}\right) b\left(s_{i}-t_{i}\right)$ and $a \geqq 0$, so we have

$$
0 \leqq a=\frac{1}{2} \sum^{n}\left(s_{i}^{*} b t_{i}+t_{i}^{*} b s_{i}\right) \leqq \frac{1}{2} \sum^{n}\left(s_{i}^{*} b s_{i}+t_{i}^{*} b t_{i}\right) .
$$

The version of Riesz-decomposition found in [8, Corollary 7.8, p. 30] shows immediately that there exists operators $z_{i}, i=1, \ldots, 2 n$ such that

$$
a=\sum^{2 n} z_{i} z_{i}^{*} \quad \text { and } \quad z_{i}^{*} z_{i} \leqq \max \left\{\left\|s_{i}\right\|^{2},\left\|t_{i}\right\|^{2}\right\} b .
$$

In order to follow the ideas from the properly infinite case we build $(I-c)$ and $(I-d)$ into pairwise orthogonal projection matrices

$$
p=\left(\begin{array}{ccccc}
(I-c) & (I-c)^{1 / 2} x & \alpha & 0 \\
x^{*}(I-c)^{1 / 2} & \vdots & x^{*} x & \beta & 0 \\
\alpha^{*} & \vdots & \beta^{*} & \gamma & 0 \\
0 & \vdots & 0 & 0 & 0
\end{array}\right), \quad q=\left(\begin{array}{ccccc}
(I-d) & (I-d)^{1 / 2} y & 0 & \lambda \\
y^{*}(I-d)^{1 / 2} & \vdots & y^{*} y & 0 & \mu \\
0 & \vdots & 0 & 0 & 0 \\
\lambda^{*} & \vdots & \mu^{*} & & \nu
\end{array}\right),
$$

and therefore we seek operators $x$ and $y$ such that $(I-c)^{1 / 2}(I-d)^{1 / 2}+x y^{*}=0$ and moreover, such that the operators inside the dotted lines are majorized by a multiple of $b \otimes I_{\mathbb{C}^{k}}$ for a suitable high $k$.

By $[7$, Proposition 1.4 .5$, p. 12] there exists an operator $u$ in $\mathscr{A}$ such that

$$
(1-c)^{1 / 2}(1-d)^{1 / 2}=u\left[(I-d)^{1 / 2}(I-c)(1-d)^{1 / 2}\right]^{1 / 4} \text { and }\|u\| \leqq\left(\frac{1}{16}\right)^{1 / 4}=\frac{1}{2}
$$

Let us write $\left[\left((I-d)^{1 / 2}(I-c)(I-d)^{1 / 2}\right]^{1 / 4}=\sum^{n} z_{i} z_{i}^{*}\right.$ with $z_{i}^{*} z_{i} \leqq N b$; then we will define $x$ and $y$ as $n \times n$ matrices over $\mathscr{A}$ by

$$
x^{*}=\left(\begin{array}{ll}
\sqrt{2} z_{1}^{*} u^{*} & \\
\sqrt{2} z_{n}^{*} u^{*} & \mathbf{0}
\end{array}\right) ; \quad y^{*}=\left(\begin{array}{cc}
-\frac{1}{\sqrt{2}} z_{1}^{*} \\
-\frac{1}{\sqrt{2}} z_{n}^{*}
\end{array}\right)
$$


and we get $x y^{*}=-(I-c)^{1 / 2}(I-d)^{1 / 2}$. Next we do choose operator $f_{1}, \ldots, f_{k}$ and $g_{1}, \ldots, g_{m}$ such that

$$
\begin{aligned}
& \sum^{k} f_{i} f_{i}^{*}=I-\sum 2 u z_{i} z_{i}^{*} u^{*}-(I-c)=c-2 \sum u z_{i} z_{i}^{*} u^{*}, \\
& \sum^{m} g_{i} g_{i}^{*}=I-\sum \frac{1}{2} z_{i} z_{i}^{*}-(I-d)=d-\frac{1}{2} \sum z_{i} z_{i}^{*}
\end{aligned}
$$

and $\sum f_{i}^{*} f_{i}+\sum g_{i}^{*} g_{i} \leqq N b$.

By construction the partial isometries $v$ and $w$ in $\mathscr{A} \otimes M_{(1+n+k+m)}$ given below have pairwise orthogonal range projections of the form $p, q$ mentioned above.

$$
v=\left(\begin{array}{cc}
(I-c)^{1 / 2} \\
\sqrt{2} z_{1}^{*} u^{*} \\
\vdots \\
\sqrt{2} z_{n}^{*} u^{*} & \\
f_{1}^{*} & \mathbf{0} \\
\vdots & \\
f_{k}^{*} & \\
0 & \vdots \\
0 & -\frac{1}{\sqrt{2}} z_{n}^{*} \\
\vdots \\
\frac{1}{\sqrt{2}} z_{n}^{*} & 0 \\
0 & \\
\vdots \\
0 \\
g_{1}^{*} \\
\vdots \\
g_{m}^{*}
\end{array}\right) ; \quad w=
$$

Let $e$ denote the matrix unit (projection) corresponding to the upper left hand corner, then since we do assume that we always may factor the situation by $3 \times 3$ matrices, any physical state is linear on the algebra generated by $e$ and any other projection.

Let $s=n+k+m+1$ and $\psi$ a physical state on $\mathscr{A} \otimes M_{s}$ which extends $\varphi$ on $\mathscr{A} \otimes I$, and let $u$ be a unitary in the compact group $\mathscr{U}$ of unitaries in $M_{s}$. For each such $u$ we get another physical state extension $\psi^{u}$ by $\psi^{u}(x)=\psi\left(\left(I \otimes u^{*}\right) x(I \otimes u)\right)$, and we find by integration over $\mathscr{U}$ with respect to the normalized Haar measure that we may as well assume that $\psi$ is $\mathscr{U}$ invariant.

The linearity of $\psi$ on $C^{*}(e, p)$ implies

$$
\begin{aligned}
\mid \psi(p)-\psi(\text { epe }) \mid & \leqq|\psi((I-e) p e)|+|\psi(p(I-e))| \\
& \leqq 2 \psi((I-e) p(I-e))^{1 / 2} .
\end{aligned}
$$

By construction $(I-e) p(I-e) \leqq K(b \otimes I)(I-e) \leqq K(b \otimes I)$ so $\psi(p)=\psi(e p e)$.

The $C^{*}$-algebra generated by epe and $M_{s}$ is on the other hand $C^{*}($ epe $) \otimes M_{s}$, and therefore the restriction of $\psi$ to this algebra is linear (Theorem 3.1) and $\mathscr{U}$ invariant. Consequently we get

$$
\psi(\text { epe })=\psi((I-c) \otimes e)=s^{-1} \psi((I-c) \otimes I)=s^{-1} \varphi(I-c) .
$$

We do of course get similar results for $q$ and $p+q$, so we have proved the linearity of $\varphi$ since $\psi(p+q)=\psi(p)+\psi(q)$. 


\section{References}

1. Aarnes, J.F.: Quasi-states on $C^{*}$-algebras. Trans. Am. Math. Soc. 149, 601-625 (1970)

2. Dixmier, J.: Les $C^{*}$-algebres et leurs representations. Paris: Gauthier-Villars 1969

3. Gleason, A.M. : Measures on closed subspaces of a Hilbert space. J. Math. Mech. 6, 885-893 (1957)

4. Gunson, J.: Physical states on quantum logics. I. Ann. Inst. Henri Poincaré A 17, 295-311 (1972)

5. Jauch, J.M.: Foundations of quantum mechanics. Reading. Mass. : Addison-Wesley 1968

6. Mackey, G.W.: Quantum mechanics and Hilbert space. Am. Math. Month. 64, 45-57 (1957)

7. Pedersen, G.K.: $C^{*}$-algebras and their automorphism groups. New York: Academic Press 1979

8. Takesaki, M.: Theory of operator algebras. I. Berlin, Heidelberg, New York: Springer 1979

Communicated by H. Araki

Received February 15, 1982 